PROCEEDINGS OF THE

AMERICAN MATHEMATICAL SOCIETY

Volume 135, Number 2, February 2007, Pages 353-363

S 0002-9939(06)08594-7

Article electronically published on August 21, 2006

\title{
STRICT CONTRACTIVITY OF THE 2-WASSERSTEIN DISTANCE FOR THE POROUS MEDIUM EQUATION BY MASS-CENTERING
}

\author{
J. A. CARRILlO, M. DI FRANCESCO, AND G. TOSCANI \\ (Communicated by Walter Craig)
}

\begin{abstract}
We show that the Euclidean Wasserstein distance between two compactly supported solutions of the one-dimensional porous medium equation having the same center of mass decays to zero for large times. As a consequence, we detect an improved $L^{1}$-rate of convergence of solutions of the one-dimensional porous medium equation towards well-centered self-similar Barenblatt profiles, as time goes to infinity.
\end{abstract}

\section{INTRODUCTION AND RESULT}

We consider the Cauchy problem for the one-dimensional porous medium equation posed on the whole real line $\mathbb{R}$ :

$$
\left\{\begin{array}{l}
u_{t}=\left(u^{m}\right)_{x x}, \quad m>1, \\
u(x, 0)=u_{0}(x),
\end{array}\right.
$$

where the initial datum $u_{0}$ is integrable, nonnegative and compactly supported. Under these assumptions, it is well known that there exists a unique weak solution $u(\cdot, t)$ of (1.1) globally defined in time. Moreover, it is also known that the profile $u(\cdot, t)$ is bounded and compactly supported at any positive time $t$. We refer to the surveys [2, 23, 19] for a good account of the literature concerning existence theory, free boundary regularity, qualitative and asymptotic properties of the solutions to the porous medium equations. Let us denote the total mass of $u_{0}$ by

$$
\int_{-\infty}^{+\infty} u_{0}(x) d x=M>0
$$

and its center of mass by

$$
x_{0}=\frac{1}{M} \int_{-\infty}^{+\infty} x u_{0}(x) d x .
$$

We recall that both quantities $M$ and $x_{0}$ are invariant with respect to time [19]. The long-time asymptotics for (1.1) are described by the family of self-similar source

Received by the editors July 27, 2005.

2000 Mathematics Subject Classification. Primary 35K65; Secondary 35B40.

Key words and phrases. Porous medium equation, Barenblatt solutions, Wasserstein distance. 
type Barenblatt-Pattle solutions

$$
U_{C}(x, t)=t^{-\frac{1}{m+1}}\left(C-\frac{m-1}{2 m(m+1)} x^{2} t^{-\frac{2}{m+1}}\right)_{+}^{\frac{1}{m-1}},
$$

where the constant $C$ must be chosen in order to match the initial mass $M$. More precisely, it is well known, for instance [19, that

$$
\left\|u(\cdot, t)-U_{C}(\cdot, t)\right\|_{L^{1}(\mathbb{R})}=o(1) \quad \text { as } t \rightarrow \infty,
$$

for any solution $u(t)$ having nonnegative initial datum in $L^{1}$. Without further assumptions on the initial datum, it has been proven in [19] that no algebraic rate of convergence can be determined in (1.2). If we restrict the class of initial data to those having finite $L^{m}$-norm and finite second moment, it is possible to obtain an improved rate of convergence [5]

$$
\left\|u(\cdot, t)-U_{C}(\cdot, t)\right\|_{L^{1}(\mathbb{R})} \leq C t^{-\alpha} \quad \text { as } t \rightarrow \infty
$$

where

$$
\alpha=\frac{2}{(m+1) \max (2, m)}
$$

which is sharp for $1<m \leq 2$.

Solutions to the porous medium equation can also be interpreted as a one parameter curve in the space of probability densities having finite second moment [18]. In this framework, it is also known that the flow map $u(\cdot, t)$ induces a 1 -Lipschitz semigroup with respect to the Euclidean Wasserstein distance [18, 7, 8]

$$
d_{2}^{2}(U, V)=\inf _{T_{\sharp} U=V} \int|x-T(x)|^{2} U(x) d x,
$$

where $U$ and $V$ are probability densities with finite second moment and where the push-forward condition $T_{\sharp} U=V$ is expressed by the relation

$$
\int_{-\infty}^{+\infty} \psi(x) V(x) d x=\int_{-\infty}^{+\infty} \psi(T(x)) U(x) d x \quad \text { for all } \psi \in C_{0}(\mathbb{R}) .
$$

More precisely, we have

$$
d_{2}\left(u_{1}(\cdot, t), u_{2}(\cdot, t)\right) \leq d_{2}\left(u_{1}(\cdot, 0), u_{2}(\cdot, 0)\right),
$$

where $u_{1}$ and $u_{2}$ are two solutions to the porous medium equation with initial data satisfying the above hypothesis. In the one-dimensional case, the above result (1.7) can be easily proven to be sharp by considering (for instance) any two solutions of the form $U_{C, x_{0}}, U_{C, x_{1}}$ with $x_{0} \neq x_{1}$, where $U_{C, x_{0}}$ is the shifted Barenblatt profile

$$
U_{C, x_{0}}(x, t)=t^{-\frac{1}{m+1}}\left(C-\frac{m-1}{2 m(m+1)}\left(x-x_{0}\right)^{2} t^{-\frac{2}{m+1}}\right)_{+}^{\frac{1}{m-1}} .
$$

Moreover, by taking as $u(t)$ in (1.3) any shifted Barenblatt profile $U_{C, x_{0}}^{\infty}$ with $x_{0} \neq 0$ in the case of $1<m \leq 2$, we achieve the optimal rate $t^{-\alpha}$ in the class of initial data where (1.3) is true. We observe that $U_{C, x_{0}}^{\infty}(\cdot, t)$ has center of mass $x_{0}$.

Fixing the center of mass by using well-centered Barenblatt profiles avoids spatial translations, and thus one would expect to improve the convergence rate towards them. This intuition coming from the spatial translation invariance of the equation has already been quantified in certain cases. As is well known, one can improve the rate of convergence towards a well-centered gaussian kernel for the heat equation by 
fixing the center of mass of the initial data. Indeed, one can fix a certain number of initial moments in order to recover a higher order asymptotic approximation for the solutions with a faster rate of convergence [13, 14, 16. Mass-centering for the heat equation also speeds up the entropy decay [11. In general, this suggests the possibility of detecting a more accurate asymptotic description, even in the nonlinear case, by fixing a suitable number of initial moments [25].

The occurrence of such a phenomenon was first established by J.L. Vázquez in [20. He proved that the support of a general solution takes the shape of the support of its corresponding shifted Barenblatt profile for large times, and that a faster convergence rate in $L^{\infty}$ towards such a profile can be obtained. In particular he recovered an optimal rate $t^{-1}$ of convergence in $L^{1}$ in the case of solutions with radial symmetry. We shall describe those results in detail later on (see Theorem 2.1 and Remark 2.4).

In the fast diffusion case, the spatial translation invariance is related to an explicit eigenvalue of the linearization of the rescaled equation in self-similar variables [9, 12. Therefore, we expect an improvement of the rate of convergence for the fast diffusion equation by mass-centering to the rate $t^{-1}$ in $L^{1}$ in the translation dominated range, which is given by the next eigenvalue of the linearization. A nearly optimal convergence rate in the fast diffusion range has been obtained in 15, 17] based on mass-centering.

In this paper we improve estimate (1.7) by mass-centering in the $1 \mathrm{D}$ porous medium equation. More precisely, we shall prove that it is possible to detect an algebraic rate of decay to zero of the 2-Wasserstein distance between any two solutions having the same center of mass. As an easy consequence of that, we get a faster algebraic $L^{1}$ rate of convergence towards well-centered Barenblatt profiles for a compactly supported solution with prescribed center of mass, than that in (1.3). However, we cannot achieve the optimal rate $t^{-1}$ of symmetric solutions provided in [20]. Our result is summarized in the following theorem.

Theorem 1.1. Let $u(x, t)$ be the solution to the Cauchy problem (1.1), where the initial datum $u_{0}$ is nonnegative, compactly supported and with unit mass. Let $x_{0}$ be the center of mass of $u_{0}$ and let $U_{C, x_{0}}$ be the shifted Barenblatt profile (1.8) with unit mass. Then, there exist two constants $\varepsilon, \delta>0$ and a positive time $t^{*}$ such that the following inequalities holds for $t \geq t^{*}$ :

$$
\begin{aligned}
& d_{2}\left(u(\cdot, t), U_{C, x_{0}}(\cdot, t)\right) \leq C_{1}(t+1)^{-\varepsilon}, \\
& \left\|u(\cdot, t)-U_{C, x_{0}}(\cdot, t)\right\|_{L^{1}(\mathbb{R})} \leq C_{2}(t+1)^{-\alpha-\delta},
\end{aligned}
$$

for some positive constants $C_{1}, C_{2}$.

We remark that the constants $C_{1}$ and $C_{2}$ in (1.10) depend on the initial mass $M$, whereas the polynomial decay rates $\varepsilon, \delta$ are independent on $M$ (see Remark 3.2).

\section{Preliminaries}

2.1. Time-dependent scaling and asymptotic behavior. Our starting point is the standard time-dependent scaling

$$
\begin{array}{lll}
u(x, t)=\alpha(t)^{-\lambda} v(y, s), & y=\alpha(t)^{-\lambda}\left(x-x_{0}\right), & s=\beta(t), \\
\alpha(t)=\left(1+\frac{t}{\lambda}\right), & \beta(t)=\lambda \log \alpha(t), & \lambda=\frac{1}{m+1},
\end{array}
$$


which transforms problem (1.1) into the Cauchy problem for the nonlinear Fokker Planck equation

$$
\left\{\begin{array}{l}
v_{s}=\left(y v+\left(v^{m}\right)_{y}\right)_{y}, \\
v(y, 0)=u_{0}(y)
\end{array}\right.
$$

The unique stationary solution to (2.2) with prescribed mass (the dependence on the mass is included in the positive constant $C_{M}$ below) is

$$
v_{M}^{\infty}(y)=\left(C_{M}-\frac{m-1}{2 m}|y|^{2}\right)_{+}^{\frac{1}{m-1}}
$$

which in the original variables $u(x, t)$ corresponds to a slight modification of the Barenblatt-Pattle profile (1.8), namely $U_{M, x_{0}}^{\infty}(x, t)=\lambda^{-\frac{1}{m-1}} U_{\bar{C}, x_{0}}(x, \alpha(t))$, where $\bar{C}=C_{M} \lambda$. Such a solution is explicitly given by the formula

$$
U_{M, x_{0}}^{\infty}(x, t)=\frac{1}{\alpha(t)^{\lambda}}\left(C_{M}-\frac{m-1}{2 m}\left(\frac{x-x_{0}}{\alpha(t)^{\lambda}}\right)^{2}\right)_{+}^{\frac{1}{m-1}} .
$$

We recall that the difference $U_{M, x_{0}}^{\infty}(x \cdot, t)-U_{\bar{C}, x_{0}}(\cdot, \alpha(t))$ decays to zero in $L^{1}$ with a faster rate than that in (1.10). This can be proven with similar arguments as in [6]. We now introduce the pressure variable $\omega=\frac{m}{m-1} u^{m-1}$. In terms of the pressure, the shifted self-similar solution (1.8) reads

$$
\begin{gathered}
\omega_{M, x_{0}}^{\infty}(x, t)=\frac{m}{m-1} \alpha(t)^{-\frac{m-1}{m+1}}\left(C_{M}-\frac{m-1}{2 m}\left(\frac{x-x_{0}}{\alpha(t)^{\lambda}}\right)^{2}\right)_{+} \\
=\frac{1}{2 \alpha(t)}\left(r(t)-\left(x-x_{0}\right)^{2}\right)_{+}, \quad r(t)=\frac{2 m}{m-1} C_{M} \alpha(t)^{2 \lambda} .
\end{gathered}
$$

Since we shall mostly work in the similarity variables $v(y, s)$, it is convenient to introduce the following rescaled pressure variable $z(y, s)=\frac{m}{m-1} v(y, s)^{m-1}$. In terms of this new variable $z$, the stationary solution (2.3) reads

$$
z^{\infty}(y)=\left(\frac{m}{m-1} C_{M}-\frac{y^{2}}{2}\right)_{+} .
$$

As mentioned in the Introduction, we recall that if the initial datum $u_{0}$ is compactly supported, so is the solution of (1.1) at any time $t>0$. Moreover, after a waiting time $t^{*}$, the support of the solution takes the shape of a connected interval (see [19]). For $t>t^{*}$, we denote the support of $u(t)$ by $[\underline{\zeta}(t), \bar{\zeta}(t)]$. Then, it is known that the curve $\{\underline{\zeta}(t)\}_{t>t^{*}}$ is strictly decreasing and the curve $\{\bar{\zeta}(t)\}_{t>t^{*}}$ strictly increasing [19]. Let us denote the support of the rescaled solution $v(s)$ by $[\underline{\eta}(s), \bar{\eta}(s)]$. The following theorem is due to Vázquez [20, 22, 21]. 
Theorem 2.1. The following properties hold as $t \rightarrow \infty$ with $r(t)$ defined by (2.5):

$$
\begin{array}{ll}
\underline{\zeta}(t)=-r(t)+x_{0}+o(1) & \bar{\zeta}(t)=r(t)+x_{0}+o(1), \\
\omega(x, t)-\omega_{x_{0}}^{\infty}(x, t)=o\left(t^{-\frac{m}{m+1}}\right) & \text { uniformly on } \mathbb{R}, \\
\omega_{x}(x, t)=-\frac{x-x_{0}}{(m+1) t}+o\left(\frac{1}{t}\right) & \text { uniformly on } \operatorname{supp} \omega(\cdot, t), \\
\omega_{x x}(x, t)=-\frac{1}{(m+1) t}+O\left(\frac{1}{t^{2}}\right) & \text { uniformly on } \operatorname{supp} \omega(\cdot, t) .
\end{array}
$$

Next we reformulate the above result in similarity variables.

Corollary 2.2. Given $c(m, M)=\frac{2 m C_{M}}{m-1}$, the following properties hold as $s \rightarrow \infty$ :

$$
\begin{array}{ll}
\underline{\eta}(s)=-c(m, M)+o(1) & \bar{\eta}(s)=c(m, M)+o(1), \\
z(y, s)-z_{x_{0}}^{\infty}(y)=o\left(e^{-s}\right) & \text { uniformly on } \mathbb{R}, \\
z_{y}(y, s)=-y+o\left(e^{-s}\right) & \text { uniformly on supp } z(\cdot, s), \\
z_{y y}(y, s)=-1+O\left(e^{-(m+1) s}\right) & \text { uniformly on supp } z(\cdot, s) .
\end{array}
$$

As a consequence of the above corollary, we state the following useful result.

Corollary 2.3. Let $v(y, s)$ be a solution to (2.2). Let $F(y, s)=\int_{-\infty}^{y} v(\xi, s) d \xi$. Then, there exists a small constant $\delta>0$ and a fixed $s_{0}>0$ such that, for any $s \geq s_{0}, i=1,2$, we have

$$
\begin{aligned}
& y \in\left[\underline{\eta}_{i}(s), \underline{\eta}_{i}(s)+\delta\right] \Rightarrow K_{0}\left(y-\underline{\eta}_{i}(s)\right)^{\frac{1}{m-1}} \leq v(y, s) \leq K_{1}\left(y-\underline{\eta}_{i}(s)\right)^{\frac{1}{m-1}}, \\
& y \in\left[\bar{\eta}_{i}(s)-\delta, \bar{\eta}_{i}(s)\right] \Rightarrow K_{0}\left(\bar{\eta}_{i}(s)-y\right)^{\frac{1}{m-1}} \leq v(y, s) \leq K_{1}\left(\bar{\eta}_{i}(s)-y\right)^{\frac{1}{m-1}} \\
& y \in\left[\underline{\eta}_{i}(s), \underline{\eta}_{i}(s)+\delta\right] \Rightarrow K_{0}\left(y-\underline{\eta}_{i}(s)\right)^{\frac{m}{m-1}} \leq F(y, s) \leq K_{1}\left(y-\underline{\eta}_{i}(s)\right)^{\frac{m}{m-1}}
\end{aligned}
$$$$
\text { where } K_{0} \text { and } K_{1} \text { are fixed positive constants independent on } s .
$$

Proof. Relation (2.13) implies

$$
z_{y y}(y, s) \geq-1+\varepsilon, \quad \text { as } s \geq s_{0},
$$

for $\varepsilon \ll 1$ and for some $s_{0}>0$. A double integration upon the interval $\left[\underline{\eta}_{i}(s), y\right]$ of the above relation (2.18) yields

$$
z(y, s)-z(\underline{\eta}(s), s) \geq(y-\underline{\eta}(s)) z_{y}(\underline{\eta}(s), s)-\frac{(1-\varepsilon)^{2}}{2}(y-\underline{\eta}(s))^{2} .
$$

We then use (2.10) and (2.12) to obtain, for $s \geq s_{0}$,

$$
z(y, s) \geq(y-\underline{\eta}(s))\left[c(m, M)+o(1)-\frac{(1-\varepsilon)^{2}}{2}(y-\underline{\eta}(s))\right] .
$$

By choosing $\delta>0$ small enough, we then obtain $z(y, s) \geq O(1)(y-\eta(s))$ as $|\eta(s)-y| \leq \delta$, and the first inequality in (2.14) is proven. The second inequality in (2.14) and the two inequalities in (2.15) can be proven in a similar fashion. We skip the details about these computations. The inequalities in (2.16) and (2.17) easily follow from (2.14) and (2.14) after integration in $(-\infty, y]$. 
Remark 2.4 ( $L^{1}$-rates of convergence in the literature). As observed in [20, (2.7) together with (2.6) imply

$$
\left\|u(t)-U_{M, x_{0}}^{\infty}(t)\right\|_{L^{1}(\mathbb{R})}=o\left(t^{-\gamma}\right), \quad \gamma=\frac{2}{(m+1) \max (2,2(m-1))} .
$$

We observe that, in the case $1<m \leq 2$, the above estimate improves (1.3) without providing a better rate. An explicit faster rate of convergence is obtained in 20, 19] in the case of solutions with symmetric initial datum, i.e. $u_{0}\left(x_{0}-x\right)=u_{0}\left(x_{0}+x\right)$, namely

$$
\left\|u(t)-U_{M, x_{0}}^{\infty}(t)\right\|_{L^{1}(\mathbb{R})}=O\left(t^{-1}\right) .
$$

We observe that such a rate of convergence can be heuristically recovered by means of spectral analysis of the linearized nonlinear Fokker-Planck equation (2.2) around the stationary state (see [1, 25]).

2.2. One-dimensional Wasserstein distance. Let $U_{1}$ and $U_{2}$ be two probability densities on $\mathbb{R}$ having compact support. The Euclidean Wasserstein distance between $U_{1}$ and $U_{2}$ can be analytically expressed as follows (the following procedure can be performed only in one space dimension). Consider the primitive variables

$$
F_{i}(x)=\int_{-\infty}^{x} U_{i}(\xi) d \xi, \quad i=1,2,
$$

and their pseudo-inverses $F_{i}^{-1}:[0,1] \rightarrow \mathbb{R}, F_{i}^{-1}(\rho)=\inf \{\omega \in \mathbb{R}: F(\rho)>\omega\}$, $i=1,2$. Then, the following identity is satisfied (see [24, 8, 3]):

$$
d_{2}\left(U_{1}, U_{2}\right)=\left[\int_{0}^{1}\left(F_{1}^{-1}(\rho)-F_{2}^{-1}(\rho)\right)^{2} d \rho\right]^{1 / 2}
$$

2.3. Two useful inequalities. We now recall a weighted Poincaré inequality we will need in the sequel (see the paper [10] for the proof).

Theorem 2.5 (Weighted Poincaré inequalities). Let $\mu$ and $w$ be two nonnegative measurable functions on $[a, b]$ which are finite almost everywhere, and let $\nu$ be a nonnegative finite Borel measure on $[a, b]$, with $\nu[a, b]>0$. Let $1<p \leq q<\infty$, with conjugate exponents $p^{\prime}$ and $q^{\prime}$ respectively, and define

$$
\begin{aligned}
K_{p, q}(\mu, \nu, w)= & \frac{1}{\nu[a, b]}\left(\sup _{a<x<b}\left\{\mu[x, b]^{1 / q}\left(\int_{a}^{x} \nu[a, t]^{p^{\prime}} w(t)^{1-p^{\prime}} d t\right)^{1 / p^{\prime}}\right\}\right. \\
& \left.+\sup _{a<x<b}\left\{\mu[a, x]^{1 / q}\left(\int_{x}^{b} \nu[t, b]^{p^{\prime}} w(t)^{1-p^{\prime}} d t\right)^{1 / p^{\prime}}\right\}\right) .
\end{aligned}
$$

Then,

$$
\left(\int_{a}^{b}\left|f(x)-\frac{1}{\nu[a, b]} \int_{a}^{b} f d \nu\right|^{q} \mu(x) d x\right)^{1 / q} \leq C\left(\int_{a}^{b}\left|f^{\prime}(x)\right|^{p} w(x) d x\right)^{1 / p}
$$

for all absolutely continuous functions $f$ on $[a, b]$ if and only if $K_{p, q}<\infty$. Moreover, the smallest possible constant $C$ in (2.22) satisfies the estimate

$$
\frac{K_{p, q}}{2} \leq C \leq\left(1+q / p^{\prime}\right)^{1 / q}\left(1+p^{\prime} / q\right)^{1 / p^{\prime}} K_{p, q} .
$$


We shall also make use of the following interpolation inequality involving $L^{1}$ norm and $d_{2}$-distance, which is an easy consequence of the generalized HWI inequality (Theorem 2.1) in [4, of the results in [5, 18, about the decay of the relative Fisher information and of the generalized Csiszár-Kullback inequality proven in [5].

Theorem 2.6 (Interpolation inequality). Let $u_{0} \in L_{+}^{1}(\mathbb{R}) \cap L^{\infty}(\mathbb{R})$ with second moment bounded and let $v^{\infty}$ be as in (2.3). Then, there exists a positive constant $C$ depending only on $u_{0}$ such that the solution $v(s)$ to (2.2) satisfies

$$
\left\|v(s)-v^{\infty}\right\|_{L^{1}(\mathbb{R})}^{\max (2, m)} \leq C d_{2}\left(v(s), v^{\infty}\right) e^{-2 s} .
$$

\section{Proof of Theorem 1.1}

We use the rescaled formulation (2.2) of problem (1.1). We suppose the initial datum $u_{0}$ to be compactly supported with unit mass and zero center of mass. As in the case of (1.1), the solution $v(s)$ to (2.2) at time $s$ maintains the same mass as the initial datum. The conservation of the zero first moment comes from the identity

$$
\frac{d}{d s} \int y v(y, s) d y=\int y\left(y v+\left(v^{m}\right)_{y}\right)_{y} d y=-\int y v(y, s) d y .
$$

Let us define the primitive function

$$
F(y, s)=\int_{-\infty}^{y} v(\xi, s) d \xi
$$

and its pseudo inverse $F^{-1}:[0,1] \rightarrow \mathbb{R}$ as in the previous section. Then, it can be easily computed that $F^{-1}(\rho, s)$ satisfies the following equation in $(0,1) \times \mathbb{R}_{+}$:

$$
\frac{\partial F^{-1}}{\partial s}=-F^{-1}-\frac{\partial}{\partial \rho}\left[\left(\frac{\partial F^{-1}}{\partial \rho}\right)^{-m}\right] .
$$

We remark that $F^{-1}$ satisfies (3.2) in the classical sense at least after a waiting time (see [3]). Let us then consider two solutions $v_{1}$ and $v_{2}$ to (2.2), both having unit mass and a zero center of mass, and let $F_{1}$ and $F_{2}$ be their corresponding primitive variables as in the above definition (3.1). Due to (2.20), we compute the $L^{2}$ norm of the difference $F_{1}^{-1}-F_{2}^{-1}$ as follows (see also [8, [3]):

$$
\begin{aligned}
& \frac{d}{d s} \int_{0}^{1}\left[F_{1}^{-1}(\rho, s)-F_{2}^{-1}(\rho, s)\right]^{2} d \rho=2 \int_{0}^{1}\left[F_{1}^{-1}-F_{2}^{-1}\right] \partial_{s}\left[F_{1}^{-1}-F_{2}^{-1}\right] d \rho \\
& \quad=-2 \int_{0}^{1}\left[F_{1}^{-1}-F_{2}^{-1}\right]^{2} d \rho-2 \int_{0}^{1}\left(F_{1}^{-1}-F_{2}^{-1}\right) \partial_{\rho}\left[\left(F_{1, \rho}^{-1}\right)^{-m}-\left(F_{2, \rho}^{-1}\right)^{-m}\right] d \rho
\end{aligned}
$$

Then, integration by parts in the last term of (3.3) yields

$$
\begin{aligned}
& \frac{d}{d s} \int_{0}^{1}\left[F_{1}^{-1}-F_{2}^{-1}\right]^{2} d \rho=-2 \int_{0}^{1}\left[F_{1}^{-1}-F_{2}^{-1}\right]^{2}-\left.2\left(F_{1}^{-1}-F_{2}^{-1}\right)\left(\widetilde{v}_{1}-\widetilde{v}_{2}\right)\right|_{\rho=0} ^{\rho=1} \\
& (3.4) \\
& \quad+2 \int_{0}^{1}\left(F_{1, \rho}^{-1}-F_{2, \rho}^{-1}\right)\left(\widetilde{v}_{1}^{m}-\widetilde{v}_{2}^{m}\right) d \rho=: I+B+J,
\end{aligned}
$$

where we have denoted

$$
\widetilde{v}_{i}(\rho, s)=v_{i}\left(F_{i}^{-1}(\rho, s), s\right)=\left[\partial_{\rho} F_{i}^{-1}(\rho, s)\right]^{-1}, \quad i=1,2 .
$$


The boundary term $B$ above disappears because of the relation

$$
\left(\widetilde{v}_{1}-\widetilde{v}_{2}\right)=0 \text { at } \rho=0,1
$$

and because of the finite speed of propagation property of the support of $v$, which implies that $F_{1}^{-1}$ and $F_{2}^{-1}$ are bounded at any time (see also [8]). We now consider the term

$$
J=2 \int_{0}^{1}\left(F_{1, \rho}^{-1}-F_{2, \rho}^{-1}\right)\left(\widetilde{v}_{1}^{m}-\widetilde{v}_{2}^{m}\right) d \rho=2 \int_{0}^{1}\left(F_{1, \rho}^{-1}-F_{2, \rho}^{-1}\right)\left(\left(F_{1, \rho}^{-1}\right)^{-m}-\left(F_{2, \rho}^{-1}\right)^{-m}\right) d \rho .
$$

Since the function $\phi(u)=u^{m}$ is strictly increasing, then the integrand in $J$ is not positive at any $(\rho, s)$. Therefore, as a first step (see [3]) we obtain exponential decay for $d_{2}\left(v_{1}(s), v_{2}(s)\right)$ with rate $e^{-s}$, and the contraction property (1.7) when turning back to the original variables $u, x, t$. Our intention now is to handle the term $J$ in order to recover a better exponential rate for $d_{2}\left(v_{1}(s), v_{2}(s)\right)$. To this aim, let us introduce the following notation:

$$
\begin{aligned}
& J=2 \int_{0}^{1}\left(F_{1, \rho}^{-1}-F_{2, \rho}^{-1}\right)\left(\widetilde{v}_{1}^{m}-\widetilde{v}_{2}^{m}\right) d \rho=-2 \int_{0}^{1}\left[F_{1, \rho}^{-1}-F_{2, \rho}^{-1}\right]^{2} \Sigma(\rho, s), \\
& \Sigma(\rho, s)=\left\{\begin{array}{lc}
{\left[\frac{\widetilde{v}_{1}^{m}-\widetilde{v}_{2}^{m}}{\widetilde{v}_{1}-\widetilde{v}_{2}} \widetilde{v}_{1} \widetilde{v}_{2}\right](\rho, s)} & \text { if } \widetilde{v}_{1} \neq \widetilde{v}_{2}, \\
m \widetilde{v}_{1}^{m+1} & \text { if } \widetilde{v}_{1}=\widetilde{v}_{2},
\end{array}\right. \\
& \operatorname{supp}\left(v_{i}(s)\right)=\left(\underline{\eta}_{i}(s), \bar{\eta}_{i}(s)\right), \quad i=1,2 .
\end{aligned}
$$

Proposition 3.1. The weight function $\Sigma(\rho, s)$ satisfies the following properties:

(i) $\Sigma(0, s)=\Sigma(1, s)=0$ for all $s \geq 0$.

(ii) $\Sigma(\rho, s)>0$ for all $0<\rho<1, s \geq 0$.

(iii) There exist a small positive constant $\rho_{0} \ll 1$ and $a s_{0}>0$ such that, for any $s \geq s_{0}, i=1,2$, we have

$$
\begin{aligned}
& \rho \in\left[0, \rho_{0}\right] \Rightarrow C_{0} \rho^{\frac{m-1}{m}} \leq F_{i}^{-1}(\rho, s)-\underline{\eta}_{i}(s) \leq C_{1} \rho^{\frac{m-1}{m}}, \\
& \rho \in\left[1-\rho_{0}, 1\right] \Rightarrow C_{0}(1-\rho)^{\frac{m-1}{m}} \leq F_{i}^{-1}(\rho, s)-\bar{\eta}_{i}(s) \leq C_{1}(1-\rho)^{\frac{m-1}{m}}, \\
& \Sigma(\rho, s) \geq C[\rho(1-\rho)]^{\frac{m+1}{m}}=: \sigma(\rho),
\end{aligned}
$$

where $C, C_{0}$ and $C_{1}$ are fixed positive constants independent on $s$.

Proof. Let us start with (i). By definition of $\widetilde{v}_{i}$ we easily obtain

$$
\widetilde{v}_{i}(0, s)=v_{i}\left(\underline{\eta}_{i}(s), s\right)=0, \quad \widetilde{v}_{i}(1, s)=v_{i}\left(\bar{\eta}_{i}(s), s\right)=0, \quad i=1,2 .
$$

We then compute

$$
\lim _{\rho \searrow 0} \frac{\widetilde{v}_{1}^{m}-\widetilde{v}_{2}^{m}}{\widetilde{v}_{1}-\widetilde{v}_{2}}=m \lim _{\rho \searrow 0} \xi^{m-1},
$$

for some $\xi$ belonging in the interval with extremals $\widetilde{v}_{1}(\rho, s)$ and $\widetilde{v}_{2}(\rho, s)$. Hence, the limit in (3.9) equals zero. This fact, together with (3.8), proves (i). In order to prove (ii) we only need to observe that the function of two nonnegative real variables

$$
(x, y) \in \mathbb{R}_{+} \times \mathbb{R}_{+} \longrightarrow \begin{cases}\frac{x^{m}-y^{m}}{x-y} & \text { if } x \neq y, \quad m>1 \\ m x^{m-1} & \text { if } x=y\end{cases}
$$


is always nonnegative and equals zero if and only if $x=y=0$. Therefore, the weight function $\Sigma(\rho, s)$ is zero if and only if both $\widetilde{v}_{1}$ and $\widetilde{v}_{2}$ are zero, and this occurs if and only if $\rho \in\{0,1\}$. The proof of (iii) is a straightforward consequence of (2.16) and (2.17) in Corollary 2.3, Moreover, as a simple consequence of (ii) and (iii), we have the following inequalities for $i=1,2$ and for $s \geq s_{0}$ :

$$
\widetilde{v}_{i}(\rho, s) \geq O(1) \rho^{\frac{1}{m}} \text { as } \rho \in\left[0, \rho_{0}\right] ; \widetilde{v}_{i}(\rho, s) \geq O(1)(1-\rho)^{\frac{1}{m}} \text { as } \rho \in\left[1-\rho_{0}, 1\right] .
$$

Moreover, by means of the limiting relation (2.11) together with (3.5) and (3.6) and because $F_{i}^{-1}$ are nondecreasing w.r.t. $\rho$, we can easily deduce that

$$
\widetilde{v}_{i}(\rho, s) \geq C, \quad \text { as } \rho \in\left[\rho_{0}, 1-\rho_{0}\right], \quad i=1,2,
$$

where $C$ is a fixed constant independent of $s$. Hence, from (3.11), (3.10) one can find a suitable fixed constant $C$ such that

$$
\widetilde{v}_{i} \geq C[\rho(1-\rho)]^{1 / m}, \quad i=1,2 .
$$

Finally, by means of the mean value formula

$$
\frac{\widetilde{v}_{1}(\rho, s)^{m}-\widetilde{v}_{2}(\rho, s)^{m}}{\widetilde{v}_{1}(\rho, s)-\widetilde{v}_{2}(\rho, s)}=m \xi^{m-1}
$$

for some $\xi$ belonging in the interval with extremal points $\widetilde{v}_{1}(\rho, s)$ and $\widetilde{v}_{2}(\rho, s)$, we can use the lower estimate (3.12) in the definition of $\Sigma$, and this proves the desired estimate (3.7).

By combining (3.7) and (3.4), we obtain

$$
\frac{d}{d s} \int_{0}^{1}\left[F_{1}^{-1}-F_{2}^{-1}\right]^{2} d \rho \leq-2 \int_{0}^{1}\left[F_{1}^{-1}-F_{2}^{-1}\right]^{2} d \rho-2 \int_{0}^{1}\left[F_{1, \rho}^{-1}-F_{2, \rho}^{-1}\right]^{2} \sigma(\rho) d \rho .
$$

Next we exploit the weighted Poincaré inequality (2.22) in (3.13) in order to increase the exponential rate of convergence to zero of the term $d_{2}\left(v_{1}, v_{2}\right)$. Following the same notation as in Theorem 2.5, we perform the following substitutions:

$$
\begin{aligned}
& p \quad \longrightarrow 2, \quad q \quad \longrightarrow 2, \\
& \mu, \nu \quad \longrightarrow \text { Lebesgue measure, } w \longrightarrow \sigma \text {, } \\
& x \in[a, b] \quad \longrightarrow \rho \in[0,1], \quad f(\cdot) \quad \longrightarrow \quad F_{1}^{-1}(\cdot, s)-F_{2}^{-1}(\cdot, s) .
\end{aligned}
$$

Since $v(s)$ has zero first moment, the change of variable $\rho=F(y, s)$ yields

$$
\int_{0}^{1} F^{-1}(\rho, s) d \rho=\int_{-\infty}^{+\infty} y F_{y}(y, s) d y=\int_{-\infty}^{+\infty} y v(y, s) d y=0 .
$$

Hence, with the notation of Theorem 2.5. the term $\int_{a}^{b} f d \nu$ in (2.22) equals zero. We then compute the constant $K_{2,2}$ in (2.21). From simple calculations, it is easy to see that the condition

$$
\sup _{0 \leq \rho \leq 1}\left\{\rho^{1 / 2}\left(\int_{\rho}^{1}(1-t)^{1-\frac{1}{m}} t^{-1-\frac{1}{m}} d t\right)^{1 / 2}\right\}<\infty
$$

is sufficient to have $K_{2,2}<\infty$. But (3.14) is clearly satisfied for $m>1$. We can then employ (2.22), and we obtain

$$
\frac{d}{d s} \int_{0}^{1}\left[F_{1}^{-1}-F_{2}^{-1}\right]^{2} d \rho=-\left(2+2 \varepsilon_{0}\right) \int_{0}^{1}\left[F_{1}^{-1}-F_{2}^{-1}\right]^{2} d \rho,
$$


for some positive constant $\varepsilon_{0}$, and this implies

$$
\int_{0}^{1}\left[F_{1}^{-1}-F_{2}^{-1}\right]^{2} d \rho \leq C e^{-\left(2+2 \varepsilon_{0}\right) s} .
$$

By expressing (3.15) in the original variables $u, x, t$ of the porous medium equation, according to the time- dependent scaling (2.1), we obtain (1.9). Inequality (1.10) then follows from (1.9) and the interpolation inequality (2.24).

Remark 3.2. As mentioned before in the Introduction, we can prove that the decay rates $\varepsilon$ and $\delta$ in (1.10) are independent on the mass. Suppose $u$ is the solution to (1.1) with $\int_{\mathbb{R}} u_{0} d x=M$. Suppose without loss of generality that $u_{0}$ has zero center of mass. Let us define

$$
v(x, t)=\frac{1}{M} u\left(x, \frac{t}{M^{m-1}}\right) .
$$

Then, since $v$ solves the same equation and since its mass is 1 , we can apply the result in Theorem 1.1 and obtain

$$
\left\|v(\cdot, \tau)-U_{C, 0}(\cdot, \tau)\right\|_{L^{1}} \leq C_{2}(\tau+1)^{-\alpha-\delta},
$$

which can be rephrased as

$$
\left\|u(\cdot, t)-M U_{C, 0}\left(\cdot, M^{m-1} t\right)\right\|_{L^{1}} \leq C_{2} M^{1-(m-1)(\alpha+\delta)} t^{-\alpha-\delta},
$$

and this proves our assertion because the rescaled Barenblatt profile in the above formula matches with $U_{C, 0}(\cdot, t)$ faster than $t^{-\alpha-\delta}$.

\section{ACKNOWLEDGEMENTS}

This work has been partially supported by the EU-funded IHP-project 'HYKE'. The work of the second author was partially supported by the Wittgenstein 2000 Award of Peter A. Markowich. The authors would like to thank Professor Juan Luis Vázquez for useful discussions and suggestions.

\section{REFERENCES}

[1] S. Angenent, Large time asymptotics for the porous media equation, Nonlinear diffusion equations and their equilibrium states, I (Berkeley, CA, 1986), Math. Sci. Res. Inst. Publ., vol. 12, Springer, New York, 1988, pp. 21-34. MR0956056 (90k:35028)

[2] D. G. Aronson, The porous medium equation, Nonlinear diffusion problems (Montecatini Terme, 1985), Lecture Notes in Math., vol. 1224, Springer, Berlin, 1986, pp. 1-46. MR0877986 (88a:35130)

[3] J. A. Carrillo, M. P. Gualdani, and G. Toscani, Finite speed of propagation in porous media by mass transportation methods, C. R. Math. Acad. Sci. Paris 338 (2004), no. 10, 815-818. MR2059493 (2005a:35155)

[4] J. A. Carrillo, R. J. McCann, and C. Villani, Kinetic equilibration rates for granular media and related equations: entropy dissipation and mass transportation estimates, Rev. Mat. Iberoamericana 19 (2003), no. 3, 971-1018. MR2053570(2005a:35126)

[5] J. A. Carrillo and G. Toscani, Asymptotic $L^{1}$-decay of solutions of the porous medium equation to self-similarity, Indiana Univ. Math. J. 49 (2000), no. 1, 113-142. MR1777035 (2001j:35155)

[6] Long-time asymptotics for strong solutions of the thin film equation, Comm. Math. Phys. 225 (2002), no. 3, 551-571. MR1888873 (2002m:35115)

[7] J. A. Carrillo, R. J. McCann, and C. Villani, Contractions in the 2-Wasserstein length space and thermalization of granular media, to appear in Archive for Rational Mechanics and Analysis (2005). 
[8] J. A. Carrillo and G. Toscani, Wasserstein metric and large-time asymptotics of nonlinear diffusion equations, New Trends in Mathematical Physics (In Honour of the Salvatore Rionero 70th Birthday), World Scientific, Singapore, 2005, pp. 234-244. MR2163983 (2006c:35147)

[9] J. A. Carrillo and J. L. Vázquez, Fine asymptotics for fast diffusion equations, Comm. Partial Differential Equations 28 (2003), 1023-1056. MR1986060(2004a:35118)

[10] S. K. Chua and R. L. Wheeden, Sharp conditions for weighted 1-dimensional Poincaré inequalities, Indiana Univ. Math. J. 49 (2000), no. 1, 143-175. MR.1777034 (2001h:26021)

[11] D. Cordero-Erausquin and R. J. McCann, Accelerated diffusion to minimum entropy, personal communication (2005).

[12] J. Denzler and R. J. McCann, Fast diffusion to self-similarity: complete spectrum, long time asymptotics, and numerology, Arch. Rational Mech. Anal. 175 (2005), 301-342. MR2126633 (2005k:35214)

[13] J. Duoandikoetxea and E. Zuazua, Moments, masses de Dirac et décomposition de fonctions, C. R. Acad. Sci. Paris Sér. I Math. 315 (1992), no. 6, 693-698. MR1183805 (94a:42046)

[14] T. Goudon, S. Junca, and G. Toscani, Fourier-based distances and Berry-Esseen like inequalities for smooth densities, Monatsh. Math. 135 (2002), no. 2, 115-136. MR1894092 (2003d:60054)

[15] Y. J. Kim and R. J. McCann, Potential theory and optimal convergence rates in fast nonlinear diffusion, Preprint, 2004

[16] Y. J. Kim and W. M. Ni, Higher order approximations in the heat equation and the truncated moment problem, Preprint, 2005.

[17] R. J. McCann and D. Slepcev, Nearly optimal convergence rates for centered solutions to the fast-diffusion equations, Preprint, 2005.

[18] F. Otto, The geometry of dissipative evolution equations: the porous medium equation, Comm. Partial Differential Equations 26 (2001), no. 1-2, 101-174. MR.1842429 (2002j:35180)

[19] J. L. Vázquez, Asymptotic behaviour for the porous medium equation posed in the whole space, J. Evol. Equ. 3 (2005), 67-118. MR.1977429(2004d:35138)

[20] _ Asymptotic behaviour and propagation properties of the one-dimensional flow of gas in a porous medium, Trans. Amer. Math. Soc. 277 (1983), no. 2, 507-527. MR0694373 (84h:35014)

[21] , Behaviour of the velocity of one-dimensional flows in porous media, Trans. Amer. Math. Soc. 286 (1984), no. 2, 787-802. MR0760987 (86b:35016)

[22] _ The interfaces of one-dimensional flows in porous media, Trans. Amer. Math. Soc. 285 (1984), no. 2, 717-737. MR0752500 (85h:35229)

[23] _ An introduction to the mathematical theory of the porous medium equation, Shape optimization and free boundaries (Montreal, PQ, 1990), NATO Adv. Sci. Inst. Ser. C Math. Phys. Sci., vol. 380, Kluwer Acad. Publ., Dordrecht, 1992, pp. 347-389. MR1260981 (95b:35101)

[24] C. Villani, Topics in optimal transportation, Graduate Studies in Mathematics, vol. 58, American Mathematical Society, Providence, RI, 2003. MR1964483 (2004e:90003)

[25] T. P. Witelski and A. J. Bernoff, Self-similar asymptotics for linear and nonlinear diffusion equations, Stud. Appl. Math. 100 (1998), no. 2, 153-193. MR.1491842 (99d:35081)

Departament de Matemàtiques - ICREA, Universitat Autònoma de Barcelona, E08193 Bellaterra, Spain

E-mail address: carrillo@mat.uab.es

Johann Radon Institute for Computational and Applied Mathematics (RiCAM), Austrian Academy of Sciences (ÖAW), A-4040 Linz, Austria

E-mail address: marco.difrancesco@oeaw.ac.at

Current address: Sezione di Matematica per L'Ingegneria, Universita di L'Aquila, Piazzale

Pontieri, Monteluco di Roio, I-67100 L'Aquila, Italy

Dipartimento di Matematica, Università di PaVia, I-27100 Pavia, Italy

E-mail address: giuseppe.toscani@unipv.it 